\title{
Colecistectomía laparoscópica ambulatoria: revisión narrativa
}

\section{Ambulatory Laparoscopic Cholecystectomy: narrative review}

\author{
Dhayra Karem Barreto ${ }^{1}$, Jesús Eduardo Díaz ${ }^{2}$, Guillermo Julián Sarmiento² .
}

\section{RESUMEN}

La colecistectomía laparoscópica es el tratamiento aceptado para la enfermedad biliar. Actualmente se han logrado tiempos quirúrgicos más cortos y menos complicaciones perioperatorias, lo que ha llevado a considerar una estancia hospitalariapostquirúrgica cada vez más corta, llegando a cumplir con la definición de cirugía ambulatoria. Varios estudios han confirmado la seguridad relativa de la colecistectomía laparosópica ambulatoria con

\begin{abstract}
Laparoscopic cholecystectomy is the accepted treatment for biliary disease. Shorter surgical times and less perioperative complications have now been achieved, which has led to a shorter postoperative hospital stay, reaching the definition of ambulatory surgery. Several studies have confirmed the relative safety of outpatient laparoscopic cholecystectomy with low mortality and morbidity; however, concerns remain about patient
\end{abstract}

\section{Historial del artículo:}

Fecha de recepción: 20/02/2020

Fecha de aprobación: 25/05/2020

1 Universidad del Valle, Médico General. Cali, Colombia.

2 Universidad del Cauca, Departamento de Ciencias Quirúrgicas. Hospital Universitario San José, Popayán, Colombia.

Correspondencia: Jesús Eduardo Díaz. Dirección: Carrera 6 \# 10N-142. Departamento de Ciencias Quirúrgicas, Hospital Universitario San José, tercer piso, Popayán, Colombia. Correo Electrónico: jediaz@unicauca.edu.co

Como citar este artículo: Barreto DK, DíazJE, Sarmiento GJ. Colecistectomía laparoscópica ambulatoria: revisión narrativa. Revista de la Facultad de Ciencias de la Salud de la Universidad del Cauca. 2020;22(2):18-25. https://doi.org/10.47373/rfcs.2020.v22.1458 
baja mortalidady morbilidad; sin embargo, siguen surgiendo preocupaciones sobre la seguridad del paciente para la detección y manejo de las complicaciones posoperatorias. Se realizó una revisión de la literatura en la que se analizaron publicaciones hechas en los últimos 15 años, encontrando estudios que indican que la colecistectomía laparosópica ambulatoria es segura si se hace una selección adecuada de lospacientes, así como metaanálisis que han concluido que no hay mortalidad a corto plazo ni diferencias en morbilidad y readmisiones, al compararla con la colecistectomía laparosópica de estancia habitual. Por lo anterior, con lo encontrado en la literatura mundial, se puede considerar que es posible realizar la colecistectomía laparosópica ambulatoria de forma segura si hay una selección adecuada de pacientes junto con el cumplimiento de requisitos específicos de egreso.

Palabras clave: Colecistectomía Laparosópica, Procedimientos Quirúrgicos Ambulatorios, Colelitiasis, Morbilidad. (DeCS)

\section{INTRODUCCIÓN}

La colecistectomía laparoscópica (CL) es actualmente el tratamiento aceptado para la enfermedad biliar sintomática y sigue siendo uno de los procedimientos quirúrgicos más frecuentes $(1,2)$. Las indicaciones incluyen, pero no se limitan a colelitiasis sintomática, disquinesia biliar, colecistitis aguda, complicaciones relacionadas con cálculos biliares y pólipos en la vesícula biliar. Se debe agregar que se ha convertido en el tratamiento de elección para pacientes con colelitiasis sintomática, al ser un procedimiento seguro en comparación con la colecistectomía abierta, con múltiples beneficios para el paciente como cicatrices más pequeńas, dolor reducido, hospitalización más corta y un retorno más rápido a las actividades diarias normales y al trabajo (3). Respecto a los pacientes con pólipos en la vesícula biliar, la CL está indicada en el manejo de todos los que manifiesten sintomatología, sin importar el tamańo de la lesión, porque ha mostrado tener efecto en el alivio de los síntomas; asimismo, está indicada en pacientes con pólipos de tamańo superior a $10 \mathrm{~mm}$ por su asociación con cáncer $(4,5)$.

El objetivo de esta revisión narrativa fue analizar la factibilidad de la realización de la CL, como cirugía ambulatoria de forma segura para el paciente, y su aplicación en nuestro medio. safety for the detection and management of postoperative complications. A literature review included publications made in the last 15 years found studies that indicate ambulatory laparoscopic cholecystectomy is safe if the patients are appropriately selected, along with metaanalyzes that have concluded there is no mortality in the shortterm, no differences in morbidity and readmissions when is compared with standard-stay laparoscopic cholecystectomy. Therefore, with what is found in the world literature, it can be considered that the ambulatory laparoscopic cholecystectomy can be safely performed if there is an adequate selection of patients together with the fulfillment of specific discharge requirements.

Key words: Cholecystectomy, Laparoscopic, Ambulatory Surgical Procedures, Cholelithiasis, Morbidity. (MeSH)

\section{MÉTODOS}

Se realizó una búsqueda en las bases de datos PubMed, Embase, Science Direct, Trip Database y Google Académico de estudios originales, artículos de revisión, ensayos clínicos controlados, revisiones sistemáticas y metaanálisis; de los últimos 15 ańos, durante los meses de febrero de 2017 a mayo de 2018, en inglés y espańol. Se utilizaron como palabras clave: colecistectomía laparoscópica ambulatoria, complicaciones, seguridad del paciente.

\section{DESARROLLO Y DISCUSIÓN}

\section{Cirugía Ambulatoria}

La cirugía ambulatoria es aquella cuya estancia hospitalaria durante el período de recuperación, hasta el egreso del paciente, es menor de 12 horas (6,7); ha demostrado tener ventajas sobre la cirugía con hospitalización, entre las que se incluyen: tiempos de estancia más cortos, movilización temprana, menor riesgo de infecciones postoperatorias y aumento de la satisfacción general del paciente. También se ha demostrado que es beneficiosa para las instituciones de salud a través de significativos ahorros de costos y 
descongestión de los servicios mediante la optimización del tiempo de programación quirúrgica, disminución de días-cama y un mayor alcance de funciones para el personal auxiliar, aumentando así la satisfacción del personal de salud $(8,9)$.

\section{Colecistectomía laparoscópica realizada como cirugía ambulatoria}

A nivel mundial, es habitual dejar los pacientes hospitalizados hasta el día siguiente de la CL; sin embargo, con el refinamiento de la técnica quirúrgica y anestésica, se han logrado tiempos quirúrgicos más cortos, menos complicaciones durante y después de la cirugía, mejoría en el manejo del dolor, así como la prevención de náuseas y vómitos. Esta rápida recuperación y los excelentes resultados quirúrgicos de la CL han llevado a una estancia hospitalaria cada vez más corta (10), siendo su realización de forma ambulatoria la que en los últimos ańos ha ganado popularidad, por lo cual ahora es ampliamente practicada en Estados Unidos $(11,12)$ y se ha introducido paulatinamente en algunos países en desarrollo con infraestructuras menos desarrolladas y en hospitales con pocas unidades de servicio de hospitalización (13).

Muchos ensayos clínicos controlados han evaluado la viabilidad, seguridad y eficacia de la CL ambulatoria; sin embargo, los resultados clínicos siguen siendo inconsistentes, por lo cual se han realizado diferentes revisiones sistemáticas con los ensayos clínicos disponibles. El más reciente meta-análisis fue realizado por Huairong y cols. (14) en el que se incluyeron 1430 pacientes a partir de 12 estudios realizados en Suiza, EEUU, Espańa, Australia, Países Bajos y Reino Unido, encontrándose que no se registró mortalidad a corto plazo y no hubo diferencias en la morbilidad general, hospitalización prolongada y tasa de readmisión.

A pesar de lo anterior, siguen preocupando la seguridad del paciente en relación con la detección y manejo de las complicaciones relacionadas a la CL como la lesión de la vía biliar principal, hemorragia en el triángulo de Calot, lesión con electrocauterio que puede ocasionar estenosis de la vía biliar, la lesión de vasos de la pared abdominal o grandes vasos, lesión de víscera hueca/maciza, absceso intraabdominal, la infección de herida quirúrgica, íleo y aquellas derivadas del neumoperitoneo como: embolia gaseosa, neumotórax, problemas respiratorios y repercusiones hemodinámicas. El sangrado y la lesión del conducto biliar son las principales complicaciones graves después de la colecistectomía laparoscópica $(15,16)$ y estas generalmente se detectan en el momento de la cirugía. Uno de los estudios multicéntricos más extensos evaluó 77.604 CL realizados en 4.292 hospitales, con tasas muy bajas de complicaciones postoperatorias vitales, que se detectaron durante las primeras 8 horas. Por lo tanto, un período de observación prudente de 6-8 horas podría ser suficiente para identificar complicaciones vitales (17).

\section{Requisitos para la realización de colecistectomía laparoscópica como cirugía ambulatoria}

Una serie de principios básicos son necesarios para determinar la aplicación de un protocolo de la CL ambulatoria y asegurar la máxima seguridad para los pacientes, así como el éxito del procedimiento (18):

- Criterios de selección adecuados para pacientes que acepten este tipo de cirugía sin hospitalización.

- Técnica quirúrgica meticulosa por cirujanos entrenados en este tipo de abordaje laparoscópico.

- Análisis y prevención de complicaciones postoperatorias tempranas.

- Criterios rigurosos para aprobar el egreso temprano.

- Control estricto del postoperatorio inmediato con una serie de controles clínicos.

- Evaluación del grado de satisfacción y calidad percibida por los pacientes.

El tipo de patología de la vesícula biliar es un factor determinante para la realización de la CL ambulatoria, ya que se ha evidenciado que el engrosamiento de la pared de la vesícula biliar observado en la ecografía hepatobiliar, triplica la probabilidad de hospitalización postquirúrgica (18) y que la historia previa de colecistitis aguda o pancreatitis se ha relacionado con el fracaso del procedimiento como cirugía ambulatoria (15,19-21). Por lo anterior, se considera que los pacientes a quienes se les puede realizar CL ambulatoria son aquellos con patología no complicada de la vesícula biliar; es decir, pacientes con colelitiasis que tengan como único síntoma dolor abdominal con parámetros de laboratorio normales (22); así como a pacientes con pólipos de características benignas sin otras alteraciones asociadas (fija a la pared, que se proyecta hacia la luz y que no presenta sombra acústica, pediculados y múltiples) (4).

También son importantes los factores propios del paciente que influyen en CL ambulatoria como:

- Edad: es una de las variables independientes más importantes para el éxito de la cirugía ambulatoria, ya que tener más de 65 ańos es un factor predictivo para el fracaso en la CL ambulatoria (7,23-25). 
- Cirugía en hemiabdomen superior por la posibilidad de encontrar adherencias $(19,25)$.

- Clasificación ASA: sistema de clasificación que utiliza la American Society of Anesthesiologists para estimar el riesgo que planteala anestesiaparalos distintos estadosdel paciente, va desde ASAI que es el paciente sano hastaASAV que es el enfermo terminal. Muchos autores limitan los criterios de inclusión a los grados ASA I y II, aunque en otros estudios se incluyen pacientes estables ASA III (15,26-29).

- Consumo de anticoagulantes orales (30).

- Aceptación del procedimiento ambulatorio: presenta diferencias según el grado de información suministrada sobre el procedimiento, así como la edad, sexo y antecedentes socioculturales del paciente. Se debe detallar la información para garantizar la máxima calidad del proceso asistencial, evitando así los efectos indeseables de la falta de información (31,32).

- Nauseas/Emesis después de la CL: presenta una incidencia global entre $12-52 \%$ y puede prolongar la permanencia del paciente en un 56\%, con el consiguiente retraso o imposibilidad para el alta hospitalaria $(33,34)$.

- La variabilidad interindividual en el dolor abdominal postoperatorio es característica después de la CL: Entre el 33-50\% de los pacientes sufren dolor intenso el mismo día de la operación, lo que requiere analgesia y es responsable de hospitalizaciones luego de la intervención quirúrgica en el $24-41 \%$ de los pacientes $(35,36)$.

\section{Hospitalizaciones inesperadas y readmisiones en colecistectomía laparoscópica ambulatoria}

La tasa de hospitalizaciones inesperadas en la CL ambulatoria oscila entre 6 y $25 \%$. Esto se debe principalmente a la aparición de síntomas postoperatorios (vómitos y dolor abdominal), la conversión a cirugía abierta y la falta de seguridad del paciente para el alta temprana $(18,27,37,38)$.

Con relación a la readmisión, definida según el criterio del CMS (Centers for Medicare \& Medicaid Services) como una admisión al mismo centro médico del que se dio egreso dentro de los 30 días posteriores al alta (39), un estudio realizado por Rana et al (40) evidenció que la tasa más alta ocurrió dentro de la primera semana (50\%), seguida de la cuarta semana (22.7\%). El análisis de los datos puso de relieve que la causa más común de readmisión fue el dolor postoperatorio (a pesar de analgesia ambulatoria), seguido por causas biliares como cálculos biliares retenidos, pérdida de bilis y pancreatitis.

\section{Experiencia en Latinoamérica en colecistectomía laparoscópica ambulatoria}

En Latinoamérica diferentes grupos han reportado sus experiencias en CL ambulatoria. En Chile se reportó una serie de 357 casos en los que el $91.9 \%$ de los pacientes fueron dados de alta luego de cumplir el período de observación de 6 horas, $2 \%$ presentaron alguna causa de morbilidad y un $2.2 \%$ presentaron readmisiones (37). En México, una serie de 597 casos con una estancia postquirúrgica promedio de 7.18 horas y en el 7.38\% de los pacientes no fue exitoso el manejo ambulatorio (38). Otros países en los que se han descrito series a favor de la CL ambulatoria incluyen: Ecuador (39), Costa Rica (40) y Honduras (41).

En Colombia se han reportado tres experiencias entre Bogotá $(42,43)$ y Cartagena (44) en las que el tiempo hasta el egreso fue en promedio 7.6 horas con un rango de 3 a 10; se presentó bajo número de complicaciones, situaciones no previstas y reingresos. Los principales datos de estas experiencias se resumen en la tabla 1.

Tabla 1. Experiencias de colecistectomía laparoscópica ambulatoria en Colombia.

\begin{tabular}{|c|c|c|c|c|c|c|}
\hline $\begin{array}{l}\text { Centro médico } \\
\text { en Colombia }\end{array}$ & $\begin{array}{c}\text { Concepto CL ambu- } \\
\text { latoria }\end{array}$ & $\begin{array}{c}\text { No. } \\
\text { pacientes } \\
\text { incluidos }\end{array}$ & $\begin{array}{l}\text { No. pacientes } \\
\text { CL ambula- } \\
\text { toria exitosa } \\
\text { (\%) }\end{array}$ & $\begin{array}{c}\text { No. Com- } \\
\text { plicacio- } \\
\text { nes (\%) }\end{array}$ & $\begin{array}{l}\text { No. Hospita- } \\
\text { lizaciones } \\
(\%)\end{array}$ & $\begin{array}{c}\text { Mortalidad } \\
(\%)\end{array}$ \\
\hline $\begin{array}{l}\text { Dispensario General del } \\
\text { Ejército Bogotá }(42)\end{array}$ & Egreso el mismo día POP & 100 & $96(96)$ & $4(4)$ & No hay dato & $1(1)$ \\
\hline CCA IPS Bogotá (43) & $\begin{array}{l}\text { Egreso en }<12 \text { horas POP } \\
\text { y sólo en el día }\end{array}$ & 829 & $805(97.1)$ & $24(2.9)$ & $15(1.8)$ & 0 \\
\hline $\begin{array}{l}\text { Clínica Gestión Salud Cartage- } \\
\text { na(44) }\end{array}$ & Egreso en $<6$ horas POP & 1260 & $1179(93.6)$ & $81(6.4)$ & $23(1.8)$ & 0 \\
\hline
\end{tabular}

POP: postoperatorio 


\section{Creación de protocolos en colecistectomía laparoscópica ambulatoria}

En todos los niveles de atención en salud, los protocolos son un buen instrumento para el mantenimiento de un nivel alto de calidad, a la vez que limitan el uso inadecuado de recursos mediante la disminución de la variabilidad y sirven para detectar e identificar problemas de calidad tanto de la práctica clínica como de la continuidad asistencial entre distintos niveles del sistema, facilitando la introducción de cambios y su monitorización (45). La creación de un protocolo para la CL ambulatoria puede permitir a una institución de salud decidir su implementación de forma estandarizada teniendo en cuenta su costo-efectividad, seguridad y satisfacción en el equipo de salud y el paciente.

En la literatura hasta el momento se encuentran pocas publicaciones sobre el proceso de construcción de protocolos para la realización de colecistectomía laparoscópica ambulatoria como las de Stephenson en 1993 (46), Calland en 2001 (47), Vega en 2006 (40), Galarza en 2015 (39) y Qiu en 2016 (48). Estos protocolos fueron creados considerando la evidencia científica disponible en su momento, pero con diversas metodologías. En el trabajo de Stephenson utilizaron adicionalmente una guía de cirugía mayor ambulatoria como base para su construcción y en el de Qiu se describe ampliamente la metodología por fases, que incluye la modificación del protocolo según las discusiones realizadas con el personal involucrado en el cuidado perioperatorio del paciente.

En Brasil se desarrolló el protocolo ACERTO, basado en los principios del programa de recuperación postquirúrgica mejorada (Enhanced Recovery After Surgery-ERAS- por sus siglas en inglés) en procedimientos de cirugía mayor y menor incluyendo colecistectomía. El reporte de sus resultados, hecho por Aguilar-Nascimento (49), con la intervención de 230 pacientes incluidos 74 a los que se realizó colecistectomía (51 laparoscópica - 23 abierta), evidencia que se logró el cumplimiento del protocolo en el $52.2 \%$ de los pacientes, con disminución significativa del tiempo de ayuno, volumen de líquidos endovenosos perioperatorios, complicaciones infecciosas y no infecciosas. Respecto a las experiencias en Colombia, reportadas en la literatura (42-44), ninguna describe el proceso de construcción del protocolo que aplicaron en las instituciones ni se hace mención de un proceso de prueba piloto o período de transición para su implementación.

La transición del hospital al hogar puede exponer a los pacientes a eventos adversos durante el período posterior al alta, por lo cual un proceso de egreso estandarizado y basado en evidencia es crítico para que se pueda hacer de forma segura y prevenir readmisiones evitables (50). La ausencia de criterios objetivos de elegibilidad y de herramientas basadas en la evidencia para la realización de CL ambulatoria puede incrementar el riesgo en pacientes con condiciones que los hacen susceptibles a eventos adversos e incluso excluir pacientes para los cuales esta estrategia podría ser beneficiosa.

\section{CONCLUSIONES}

Es posible realizar CL ambulatoria de forma segura si se hace una selección adecuada de pacientes según edad, antecedentes farmacológicos, clasificación ASA, tipo de patología biliar, dolor postoperatorio y presencia de náuseas/emesis, junto con el cumplimiento de requisitos específicos de egreso. Sin embargo, hace falta la realización de un mayor número de estudios que permitan establecer si los resultados reportados en la literatura mundial son aplicables a nuestro medio.

Colombia, siendo un país en desarrollo, no es ajeno a los problemas operativos del sistema de salud, tales como un número limitado de camas y quirófanos disponibles para atender las necesidades en salud de sus habitantes. Se han encontrado algunas experiencias con la CL ambulatoria en Colombia descritas en la literatura que han evidenciado altas tasas de éxito y frecuencias bajas de complicaciones y mortalidad; sin embargo, no se encontraron protocolos estandarizados. En este sentido, se considera que el desarrollo de protocolos que permitan aplicar de forma segura y estandarizada estrategias para ampliar la cobertura en salud, incluyendo el manejo quirúrgico ambulatorio de enfermedades como la colelitiasis, es un objetivo deseable y necesario.

\section{CONTRIBUCIÓN DE LOS AUTORES}

Dhayra Karem Barreto redactó el artículo.

Guillermo Julián Sarmiento y Jesús Eduardo Díaz editaron el artículo y aprobaron la versión final.

\section{CONFLICTO DE INTERESES}

Ninguno de los autores declara conflicto de intereses.

\section{FINANCIACIÓN}

Ninguna fuente de financiación. 


\section{REFERENCIAS}

1. Begos DG, Modlin IM. Laparoscopic cholecystectomy: From gimmick to gold standard. J Clin Gastroenterol. 1994;19(4):325-30. https://doi.org/10.1097/00004836199412000-00015

2. Sain AH. Laparoscopic cholecystectomy is the current \&quot;gold standard\&quot; for the treatment of gallstone disease. Ann Surg. 1996;224(5):689-90. https:// doi.org/10.1097/00000658-199611000-00019

3. Gadacz TR, Talamini MA. Traditional versus laparoscopic cholecystectomy. Am J Surg. 1991;161(3):336-8. https:// doi.org/10.1016/0002-9610(91)90591-Z

4. Colecchia A, Larocca A, Scaioli E, Bacchi-Reggiani ML, Di Biase AR, Azzaroli F, et al. Natural History of Small Gallbladder Polyps Is Benign: Evidence from a Clinical and Pathogenetic Study. Am J Gastroenterol. 2009;104(3):624-9. https://doi.org/10.1038/ajg.2008.99

5. Pejić MA, Milić DJ. [Surgical treatment of polypoid lesions of gallbladder]. Srp Arh Celok Lek. 2003;131(7-8):319-24. https://doi.org/10.2298/SARH0308319P

6. Jiménez Fuertes M, Costa Navarro D. Colecistectomía laparoscópica ambulatoria y control del dolor postoperatorio: presentación de una serie de 100 casos. Cirugía Espańola. 2015;93(3):181-6. https://doi. org/10.1016/j.ciresp.2013.04.026

7. Planells Roig M, Garcia Espinosa R, Cervera Delgado M, Navarro Vicente F, Carrau Giner M, Sanahuja Santafé Á, et al. Colecistectomía laparosópica ambulatoria. Estudio de cohortes de 1.600 casos consecutivos. Cirugía Espańola. 2013;91(3):156-62.https://doi.org/10.1016/j.ciresp.2012.08.009

8. Chauhan A, Mehrotra M, Bhatia PK, Baj B, Gupta AK. Day Care Laparoscopic Cholecystectomy: A Feasibility Study in a Public Health Service Hospital in a Developing Country. World J Surg. 2006;30(9):1690-5. https://doi. org/10.1007/s00268-006-0023-7

9. Anderson T, Walls M, Canelo R. Day case surgery guidelines. Surg. 2017;35(2):85-91. https://doi. org/10.1016/j.mpsur.2016.11.013

10. Calland JF, Tanaka K, Foley E, Bovbjerg VE, Markey DW, Blome S, et al. Outpatient laparoscopic cholecystectomy: patient outcomes after implementation of a clinical pathway. Ann Surg. 2001;233(5):704-15. https://doi. org/10.1097/00000658-200105000-00015

11. Jain PK, Hayden JD, Sedman PC, Royston CMS, O'Boyle CJ. A prospective study of ambulatory laparoscopic cholecystectomy: Training economic, and patient benefits. Surg Endosc. 2005;19(8):1082-5. https://doi. org/10.1007/s00464-004-2170-y
12. Cassinotti E, Colombo EM, Di Giuseppe M, Rovera F, Dionigi G, Boni L. Current indications for laparoscopy in day-case surgery. Int J Surg. 2008;6: S93-6. https:// doi.org/10.1016/j.ijsu.2008.12.012

13. Bal S, Reddy LGS, Parshad R, Guleria R, Kashyap L. Feasibility and safety of day care laparoscopic cholecystectomy in a developing country. Postgrad Med J. 2003;79(931):284-8. https://doi.org/10.1136/pmj.79.931.284

14. Tang H, Dong A, Yan L. Day surgery versus overnight stay laparoscopic cholecystectomy: A systematic review and meta-analysis. Dig Liver Dis. 2015;47(7):556-61. https:// doi.org/10.1016/j.dld.2015.04.007

15. Keulemans Y, Eshuis J, de Haes H, de Wit LT, Gouma DJ. Laparoscopic cholecystectomy: day-care versus clinical observation. Ann Surg. 1998;228(6):734-40. https://doi. org/10.1097/00000658-199812000-00003

16. Shamiyeh A, Wayand W. Laparoscopic cholecystectomy: early and late complications and their treatment. Langenbeck's Arch Surg. 2004;389(3):164-71. https:// doi.org/10.1007/s00423-004-0470-2

17. Strasberg SM, Hertl M, Soper NJ. An analysis of the problem of biliary injury during laparoscopic cholecystectomy. J Am Coll Surg. 1995;180(1):101-25.

18. Bueno Lledó J, Granero Castro P, Gomez i Gavara I, Ibańez Cirión JL, López Andújar R, García Granero E. Veinticinco ańos de colecistectomía laparoscópica en régimen ambulatorio. Cirugía Espańola. 2016;94(8):42941. https://doi.org/10.1016/j.ciresp.2015.03.012

19. Reddick EJ, Olsen D0. Outpatient laparoscopic laser cholecystectomy. Am J Surg. 1990;160(5):485-7. https:// doi.org/10.1016/S0002-9610(05)81009-8

20. Simpson JP, Savarise MT, Moore J. Outpatient laparoscopic cholecystectomy: what predicts the need for admission? Am Surg. 1999;65(6):525-8

21. Planells Roig M, García Espinosa R, Anaya Reig P, López Martínez C, Ballester C, Serralta Serra A, et al. Factores predictivos de colecistectomía laparoscópica dificultosa. Cirugía Espańola. 1999; 65(1):48-53.

22. Piero Portincasa, Wang DQ-H. Gallstones. In: Podolsky DK, Camilleri M, Fitz JG, Kalloo AN, Shanahan F, Wang TC. Yamada's Textbook of Gastroenterology. 6 Ed. EEUU: Wiley-Blackwell; 2016. p. 1820.

23. Zegarra RF, Saba AK, Peschiera JL. Outpatient laparoscopic cholecystectomy: safe and cost effective? Surg Laparosc Endosc. 1997;7(6):487-90. https://doi. org/10.1097/00019509-199712000-00012 
24. Bueno Lledó J, Planells Roig M, Arnau Bertomeu C, Sanahuja Santafé A, Oviedo Bravo M, García Espinosa $\mathrm{R}$, et al. Outpatient laparoscopic cholecystectomy: a new gold standard for cholecystectomy. Rev Esp Enferm Dig. 2006;98(1):14-24. https://doi.org/10.4321/S113001082006000100003

25. Voyles CR, Berch BR. Selection criteria for laparoscopic cholecystectomy in an ambulatory care setting. Surg Endosc. 1997;11(12):1145-6. https://doi.org/10.1007/ s004649900556

26. Bringman S, Anderberg B, Heikkinen T, Rn Nyberg B, Peterson E, Hansen K, et al. Outpatient laparoscopic cholecystectomy. A prospective study with 100 consecutive patients. Ambul Surg. 2001; 9:83-6. https:// doi.org/10.1016/S0966-6532(01)00076-2

27. Ammori BJ, Davides D, Vezakis A, Martin IG, Larvin M, Smith S, et al. Day-case laparoscopic cholecystectomy: a prospective evaluation of a 6-year experience. J Hepatobiliary Pancreat Surg. 2003;10(4):303-8. https:// doi.org/10.1007/s00534-002-0807-6

28. Leeder PC, Matthews T, Krzeminska K, Dehn TCB. Routine day-case laparoscopic cholecystectomy. $\mathrm{Br}$ J Surg. 2004;91(3):312-6. https://doi.org/10.1002/ bjs. 4409

29. Vuilleumier H, Halkic N. Laparoscopic Cholecystectomy as a Day Surgery Procedure: Implementation and Audit of 136 Consecutive Cases in a University Hospital. World J Surg. 2004;28(8):737-40. https://doi.org/10.1007/ s00268-004-7376-6

30. Papaceit J, Olona M, Ramón C, García-Aguado R, Rodríguez R, Rull M. Encuesta nacional sobre manejo preoperatorio y criterios de selección de pacientes en las unidades de cirugía mayor ambulatoria espańolas. Gac Sanit. 2003;17(5):384-92. https://doi.org/10.1016/ S0213-9111(03)71774-9

31. Mira J, Buil J, Lorenzo S, Vitaller J, Aranaz J. Marketing sanitario y calidad asistencial: reflexiones para el diseńo de los servicios quirúrgicos. Cirugía Espańola. 2000; 67:180-3.

32. Martínez Rodenas F, Arganda Iglesias L, Giralt Murillo C, Moreno Solórzano J, Codina Grifell J, Bueno López A, et al. Colecistectomía laparoscópica ambulatoria. Dificultades para su implementación y desarrollo. Cirugía Mayor Ambulatoria. 2014;19(2):35-42.

33. Bisgaard T, Klarskov B, Rosenberg J, Kehlet H. Factors determining convalescence after uncomplicated laparoscopic cholecystectomy. Arch Surg. 2001;136(8):917-21. https://doi.org/10.1001/ archsurg.136.8.917
34. Lehmann HP, Fleisher LA, Lam J, Frink BA, Bass EB. Patient preferences for early discharge after laparoscopic cholecystectomy. Anesth Analg. 1999;88(6):1280-5. https://doi.org/10.1097/00000539-199906000-00015

35. Joris J, Thiry E, Paris P, Weerts J, Lamy M. Pain after laparoscopic cholecystectomy: characteristics and effect of intraperitoneal bupivacaine. Anesth Analg. 1995;81(2):379-84. https://doi.org/10.1097/00000539199508000-00029

36. Pasqualucci A, de Angelis V, Contardo R, Colň F, Terrosu G, Donini A, et al. Preemptive analgesia: intraperitoneal local anesthetic in laparoscopic cholecystectomy. A randomized, double-blind, placebo-controlled study. Anesthesiology. 1996;85(1):11-20. https://doi. org/10.1097/00000542-199607000-00003

37. Pattillo JC, Kusanovic R, Salas P, Reyes J, GarcíaHuidobro I, Sanhueza M, et al. [Outpatient laparoscopic cholecystectomy. Experience in 357 patients]. Rev Med Chil. 2004;132(4):429-36. https://doi.org/10.4067/ S0034-98872004000400004

38. López Espinosa G, Zavalza González JF, Paipilla Monroy 0, Lee Rojo S. Colecistectomías laparoscópicas realizadas en Unidad de Cirugía Ambulatoria. Cir Gen. 2011;33(2):104-10.

39. Galarza DP. Evidencias en Cirugía Ambulatoria: Colecistectomía Laparoscópica con tres puertos en pacientes con Colelitiasis no complicada y Pólipos Vesiculares. Estudio desarrollado en el Centro de Atención Ambulatoria "El Batán", del IESS, de la Ciudad de Quito, durante el periodo de Julio 2011 a Julio 2012 [Especialista en Cirugía General]. Universidad Nacional de Loja; 2013.

40. Vega-Vega, Marlen; Orlich-Carranza, Claudio And Gomez-Hernandez K. Colecistectomía laparoscópica ambulatoria: Reporte preliminar de 100 casos. Acta Med Costarric. 2006;48(3):119-22.

41. Blanco Raudales E, Sierra R, Alger J. Características de colecistectomía laparosópica ambulatoria y hospitalaria, Instituto Hondureńo de Seguridad Social, Tegucigalpa, Honduras, 2012-2013. Rev Med Hondur. 2015;83(1/2):1822.

42. Zuluaga LA, Clavijo W, Tavera A. Colecistectomía laparoscópica ambulatoria en una unidad quirúrgica no hospitalaria. Rev colomb cir. 2000;2-7.

43. Moore J, Rodríguez S, Roa A, Girón M, Sanabria A, Rodríguez P, et al. Colecistectomía laparoscópica ambulatoria: modelo de programa costo-eficiente de cirugía laparoscópica. Rev Colomb Cirugía. 2004;19(1):43-53. 
44. Acuńa J, Bonfante J, Galeano N, Carrasquilla R, Manjarrez A, Alcalá R. Colecistectomía laparoscópica con manejo ambulatorio ultracorto. Rev Colomb Cirugía. 2015; 30:119-124

45. Martín Zurro A, Cano Pérez JF. Atención primaria. Concepto, organización y práctica clínica. $6^{\circ}$. Elsevier. 2008. 478-487 p.

46. Stephenson BM, Callander C, Sage M, Vellacott KD. Feasibility of "day case" laparoscopic cholecystectomy. Ann R Coll Surg Engl. 1993;75(4):249-51.

47. Calland JF, Tanaka K, Foley E, Bovbjerg VE, Markey DW, Blome S, et al. Outpatient laparoscopic cholecystectomy: Patient outcomes after implementation of a clinical pathway. Ann Surg. 2001;233(5):704-15. https://doi. org/10.1097/00000658-200105000-00015

48. Qiu C, Rinehart J, Nguyen VT, Cannesson M, Morkos A, LaPlace D, et al. An Ambulatory Surgery Perioperative Surgical Home in Kaiser Permanente Settings. Anesth Analg. 2017;124(3):768-74. https://doi.org/10.1213/ ANE.0000000000001717

49. de Aguilar-Nascimento JE, Bicudo-Salomăo A, Caporossi C, Silva RM, Cardoso EA, Santos TP. Enhancing surgical recovery in Central-West Brazil: The ACERTO protocol results. E Spen Eur E J Clin Nutr Metab. 2008;3(2): e7883. https://doi.org/10.1016/j.eclnm.2008.01.003

50. Soong C, Daub S, Lee J, Majewski C, Musing E, Nord P, et al. Development of a checklist of safe discharge practices for hospital patients. J Hosp Med. 2013;8(8):444-9. https://doi.org/10.1002/jhm.2032 